\title{
Eficacia de los comités de convivencia laboral como instrumento de prevención del acoso laboral en las medianas empresas del municipio de Girardot
}

\section{Work coexistence committee efficacy as a tool of prevention of work harassment in medium- level enterprises in Girardot municipality}

\author{
DOI: https://doi.org/10.17981/juridcuc.17.1.2021.20
}

Fecha de Recepción: 2020/02/04 Fecha de Aceptación: 2021/01/01

\author{
Julián Andrés Gaitán Reyes \\ Universidad Uniminuto. Girardot (Colombia) \\ julgaitanreyes@gmail.com
}

\section{Luperly Palacio Rodríguez \\ Universidad Uniminuto. Girardot (Colombia) luperly052671@hotmail.com}

Para citar este artículo:

Gaitán, J. \& Palacio, L. (2021). Eficacia de los comités de convivencia laboral como instrumento de prevención del acoso laboral en las medianas empresas del municipio de Girardot. Jurídicas CUC, 17(1), 589-612. DOI: http://dx.doi.org/10.17981/juridcuc.17.1.2021.20

\section{Resumen}

El presente artículo es resultado de una investigación que tuvo como objetivo principal analizar el Comité de Convivencia Laboral como herramienta suficiente e integral para la protección y reconocimiento efectivo de los derechos de las víctimas de acoso laboral en las medianas empresas del municipio de Girardot, examinando la eficacia y la funcionalidad de esta herramienta socio-jurídica. El tipo de investigación realizada fue descriptiva con un enfoque mixto. La muestra de la investigación es intencional, con 250 participantes mayores de 18 años, de diferentes niveles de formación académica y estrato socioeconómico heterogéneo. Los instrumentos utilizados fueron: observación no participante, grupos focales, entrevistas semiestructuradas y encuesta escala Likert. Como resultado, el estudio revela un panorama de ineficacia socio-jurídica, al identificarse que los reglamentos internos de trabajo no contemplan mecanismos distintos al Comité de Convivencia Laboral para prevenir el acoso laboral, se observa que existe temor de denuncia ante Comité de Convivencia Laboral por falta de protección jurídica a la víctima, la dificultad al probar las conductas consideradas acoso laboral. La investigación permite concluir que la poca imparcialidad que podría presentarse al momento de constituir el Comité de Convivencia Laboral, así como su legitimidad se ve afectada, dado que las decisiones adoptadas favorecen al acosador. Asimismo, carecen de efectividad práctica las decisiones, en el sentido que no soluciona de fondo el conflicto entre el acosador y acosado.

Palabras clave: Comité de Convivencia Laboral; eficacia de la ley; mobbing; hostigamiento laboral; Reglamento interno de trabajo; riesgos psicosociales

\section{Abstract}

The current article is the result of a research. Its main purpose was to analyze the work coexistence committee as a useful tool for the protection and recognition of rights of victims of work harassment in enterprises of medium level in Girardot. On the other hand, the idea is to evaluate its effectiveness and function as legal instrument. The type of research was descriptive research design and mixed method as well. The sample of the study was chosen, it was about 250 participants, 18 age average, they belong to different academic background and socio-economic level. The instruments applied were: observation no participant, focus group, semi structured interviews and surveys Likert scale. As a result, the study shows and poor efficacy in terms of legal functions, when identify that work internal rules are not different from the work coexistence committee. Another fact is the fear of social denunciation with the committee, it is due to the absent of legal protection a guarantees in favor to victims and the difficulty about probing the behavior considered as a work harassment. The conclusion of the research is that the stalker of person who shows a bullying attitude has an advantage over the victim, because of the poor impartialities could be presented when building the work coexistence committee. Likewise, the solutions between the abuser and the victims are not taken effectively to solve the problem.

Keywords: Work coexistence committee; effectiveness of the law; mobbing; labor harassment; Internal work regulation; psychosocial risks 


\section{INTRODUCCIÓN}

Las transformaciones constantes de la sociedad, el crecimiento económico, las nuevas tecnologías, las formas de comunicarnos, el crecimiento de las urbes, entre otras, ha generado nuevas formas de trabajo en la sociedad y por ende nuevas modalidades de contratación laboral que instan a la prevención, protección y promoción de las condiciones de salud y seguridad en el trabajo.

En ese contexto de evolución, los progresos tecnológicos han permitido potenciar el crecimiento económico y prosperidad de una nación, impulsando lo que se ha denominado la Cuarta Revolución Industrial, la cual según Schwab (2016) se caracteriza por:

La fusión de los mundos físico, digital y biológico trascenderá aún más las limitaciones de tiempo y espacio de tal manera que se fomentará la movilidad. Uno de los desafíos de la cuarta revolución industrial será la gestión de la movilidad humana para garantizar que se obtengan todos sus beneficios mediante la alineación de los derechos y deberes soberanos con los derechos y las aspiraciones individuales, la conciliación de la seguridad nacional y humana, así como la búsqueda de maneras de mantener la armonía social mientras aumenta la diversidad (p. 68).

La celeridad de estos procesos tecnológicos ha permitido el crecimiento económico y la productividad mundial (Remes et al., 2018); sin embargo, la gestión de recurso humano reconoce que el capital humano como otro activo intangible de las organizaciones es un factor determínate del éxito o del fracaso de esta. En ese sentido la globalización y los cambios tecnológicos, presentan grandes desafíos al trabajo decente basado en la dignidad, respeto y la igualdad de oportunidades (Ramírez y Ulloa, 2020).

A pesar de los cambios sustanciales de la naturaleza del trabajo, las organizaciones siguen sin resolver problemas pretéritos relacionados con conductas como el mobbing. Tal como lo revela la Organización Internacional del Trabajo (OIT, 2017), uno de cada 10 trabajadores sufre acoso laboral, y el $75 \%$ son víctimas las mujeres. Ahora bien, en el caso colombiano las cifras de denuncias de acoso 
laboral del primer periodo del año 2018, según el Ministerio del Trabajo es de 1406 casos (Portafolio, 2018).

El hostigamiento en el trabajo habitualmente constituye un riesgo laboral para la salud física y psicológica del trabajador víctima de acoso laboral. La Ley laboral exige que todo empleador debe proteger eficazmente la vida y la salud del empleado, es por eso, por lo que el acoso laboral es una de las formas de incumplir los deberes impuestos al empleador. Contrario sensu el empleador tiene la obligación legal de prevenir tales hostigamientos o ataques.

En el año 2006, se expide en Colombia la Ley 1010, por medio de la cual se adoptan medidas para prevenir, corregir y sancionar el acoso laboral y otros hostigamientos en el marco de las relaciones de trabajo, permitiendo el desarrollo para la creación y conformación de los Comités de Convivencia Laboral, como medida primigenia para enfrentar el fenómeno de acoso laboral. Y precisamente el objetivo principal de esta investigación es analizar el Comité de Convivencia Laboral como herramienta suficiente e integral para la protección y reconocimiento efectivo de los derechos de las víctimas de acoso laboral en las medianas empresas del municipio de Girardot (Colombia), examinando la eficacia, y la funcionalidad de esta herramienta socio-jurídica.

La ausencia de investigaciones sobre la eficacia de los Comités de Convivencia Laboral motivó a indagar la problemática: ¿Cómo el Comité de Convivencia Laboral es una herramienta suficiente e integral para la protección y el resarcimiento efectivo de los derechos a las víctimas de acoso laboral, y de esta manera propiciar un ambiente de trabajo sano en las medianas empresas del municipio de Girardot?

El estudio se apoyó en el tipo de investigación descriptiva, con un enfoque mixto. La muestra de la investigación es intencional, con 250 participantes mayores de 18 años de edad, de diferentes niveles de formación académica y estrato socioeconómico heterogéneo. Los instrumentos utilizados fueron: observación no participante, focos grupales, entrevistas semiestructuradas y encuestas con escala de Likert. 
Para dar respuesta a la pregunta de investigación, primero se expone el marco analítico, donde se realiza aproximación teórica sobre la eficacia de las normas jurídicas; asimismo se abordó la delimitación conceptual del acoso laboral, con la finalidad de lograr acercamiento teórico del fenómeno desde el ámbito jurídico, para un mejor desarrollo normativo del funcionamiento del Comité de Convivencia Laboral. Segundo, se establecen los hallazgos encontrados con la utilización de los instrumentos de recolección de la información que permitieron un análisis de la poca eficacia de los Comités de Convivencia Laboral en las medianas empresas del municipio de Girardot; y por último se presentan las conclusiones.

\section{Desarrollo}

\section{Marco analítico}

\section{- Eficacia de las normas Jurídicas}

El estudio de la eficacia de las normas jurídicas tiene fundamento al permitir evaluar la realización del derecho objetivo y de los derechos subjetivos, lo cual permite inferir que, a mayor eficacia de la norma, mayor legitimidad del sistema político y mayor realización de los derechos de los ciudadanos. En otras palabras, la eficacia de la norma devine del presupuesto de garantizar la seguridad jurídica a nivel social.

De esta forma, la eficacia de una norma jurídica consiste en el nivel de cumplimiento más el nivel de aplicación por los destinatarios. Dado que lo norma jurídica trae constructos sociales que van en búsqueda de la legitimación social de derecho. Por lo tanto, una norma es "ineficaz sí, y solo si, dadas las condiciones de aplicación de la misma esta no es acatada y tampoco es aplicada la sanción prescrita" (Calvo, 2007, p. 173).

La eficacia de las normas puede ser entendida tanto en un sentido jurídico como en un sentido sociológico. Para la Corte Constitucional según Sentencia C-873 (2003), la eficacia de la norma hace relación: 
En el sentido jurídico a la producción de efectos en el ordenamiento jurídico por la norma en cuestión; es decir, a la aptitud que tiene la norma de generar consecuencias en derecho en tanto ordena, permite o prohíbe algo. Por su parte, el sentido sociológico de eficacia se refiere a la forma y el grado en que la norma es cumplida en la realidad y socialmente observable. Así, se concluye que una norma es eficaz cuando es cumplida por los obligados a respetarla y que necesariamente implica un cambio o modificación de un comportamiento o las decisiones adoptadas (num. V, inc. 3.2.2, lit. c).

En ese orden, Kelsen (1969) expresa que "un ordenamiento jurídico es considerado válido, cuando sus normas, en términos generales y a grandes rasgos, son eficaces, es decir, son fácticamente acatadas y aplicadas" (p. 224). A manera de colofón, una norma jurídica es eficaz por el nivel de cumplimiento con el nivel de aplicación de los destinatarios.

Es importante señalar que existen factores que permiten contribuir a la eficacia de la norma jurídica. Para este fin es importante tomar las categorías reseñadas por Evan (1980), las cuales permiten reconocer como la eficacia e ineficacia de la norma jurídica depende de los siguientes factores: la autoridad y prestigio del legislador, la sintonía del derecho con los valores culturales, la aplicación pragmática de las normas, la adecuación de los plazos para la aplicación de la norma, la eficacia de los órganos judiciales y administrativos en la aplicación de la normas, las consecuencias jurídicas que derivan de la protección con sanciones positivas y negativas y, finalmente, la protección de derechos y garantías de quienes pueden recibir efectos nocivos por la no aplicación de la norma.

\section{- Delimitación conceptual del Acoso Laboral}

El acoso laboral es una práctica que ha dejado de ser un fenómeno, para convertirse en una realidad de algunos trabajadores. Según cifras del Ministerio de Trabajo, en el primer semestre del 2018 se reportaron 1.406 quejas por acoso laboral (Portafolio, 2018). Cuando se comparan estas cifras con el año 2017 revelan un incremento de hasta el cincuenta por ciento de esta situación. Esta 
realidad tuvo sus primeros estudios principalmente en las disciplinas como la psicología, psiquiatría y sociología. Sin embargo, se reconoce la existencia de una relación estrecha con la seguridad y salud en el trabajo, lo que genera situaciones problemáticas, que requieren soluciones desde distintas áreas del conocimiento. En ese sentido, abundan definiciones para describir el fenómeno, en función de criterios, matices, frecuencia y conductas empleadas por cada investigador.

Al respecto, López y Vázquez (2007) identifican en su investigación dieciocho términos y definiciones a nivel de doctrina internacional concluyendo que, a pesar de existir un término reconocido mundialmente sobre este tipo de violencia en el trabajo, no existe unanimidad en la comunidad científica sobre su utilización. Por ello, es importante realizar la delimitación conceptual del acoso laboral desde el ámbito de las relaciones laborales, con aproximación teórica al ámbito jurí́dico.

Desde un acercamiento conceptual, Brodsky (1976) es el primer autor que realiza una investigación sobre el trabajador hostigado basado en la promulgación de la Ley de Condiciones de Trabajo en Suecia. La investigación se centró en la dureza de la vida del trabajador en contexto de accidentes laborales y agotamiento físico. Sin embargo, no tuvo mayor impacto en el estudio científico del mobbing por no centrarse en el acoso laboral como práctica sistemática, sino más bien estos casos fueron presentados en el contexto del trabajo excesivo y el estrés laboral que debían enfrentar los trabajadores.

Universalmente el acoso laboral es conocido como mobbing, palabra utilizada en inglés para describir el comportamiento grupal de los animales. El Etólogo Lorenz (1991) llamó mobbing a los ataques de un grupo de animales más pequeños, los cuales amenazan a un solo animal más grande. El término de mobbing fue utilizado en el ámbito laboral, a principios de la década de 1980, por el profesor Leymann (1986), quién fue el primero en difundirlo durante el Congreso sobre la Higiene y Seguridad en el trabajo de Hamburgo, describiéndolo como: 
El terror psicológico en el ámbito laboral consiste en la comunicación hostil y sin ética, dirigida de manera sistemática por uno o varios individuos contra otro, que es así arrastrando a una posición de indefensión y desvalimiento, y activamente mantenido en ella (Leymann citado por González, 2002, p. 20).

\begin{abstract}
Al respecto, Hirigoyen (2012) define el acoso laboral como:
[...] cualquier manifestación de una conducta abusiva y, especialmente, los comportamientos, palabras, actos, gestos y escritos que puedan atentar contra la personalidad, la dignidad o la integridad física o psíquica de un individuo, o que puedan poner en peligro su empleo, o degradar el clima de trabajo (p. 48).
\end{abstract}

Por su parte, Piñuel (2001) da una definición más estructurada al definir el acoso laboral como:

El continuado y deliberado maltrato verbal y modal que recibe un trabajador por parte de otro u otros, que se comportan con él cruelmente con el objeto de lograr su aniquilación o destrucción psicológica y a obtener su salida de la organización a través de diferentes procedimientos ilegales, ilícitos, o ajenos a un trato respetuoso o humanitario y que atentan contra la dignidad del trabajador. El mobbig tiene como objetivo intimidar, apocar, reducir, aplanar, amedrentar y consumir, emocional e intelectualmente a la víctima, con vistas a eliminarla de la organización o satisfacer la necesidad insaciable de agredir, controlar, y destruir que suele presentar el hostigador, que aprovecha la ocasión que le brinda la situación organizativa particular (reorganización, reducción de costes, burocratización, cambios vertiginosos, etc.) para canalizar una serie de impulsos y tendencias psicopáticas (p. 55).

En ese sentido, en términos generales, el acoso laboral está estructurado bajo las conductas reiteradas, prolongadas de acoso que atenta contra los derechos fundamentales de la dignidad humana, la salud, la integridad del trabajador o trabajadora. Es decir, son hostigamientos, ataques físicos, psicológicos que ponen en peligro su empleo degrado el ambiente de trabajo. 
En este orden, el acoso laboral no es un padecimiento, sino un conjunto de conductas dañinas e inaceptables en el lugar de trabajo, considerado un riesgo al cual está expuesto el trabajador. De ahí la necesidad socio-jurídica de buscar desarrollos legislativos que protejan al trabajador de los riesgos físicos, psicológicos a los que se enfrenta una persona en el marco del cumplimiento de un trabajo. Precisamente, la Ley 1010 (2006) crea herramientas capaces de adoptar medidas para prevenir, corregir y sancionar el acoso laboral y otros hostigamientos en el marco de las relaciones de trabajo.

De forma que, para la Sentencia T-882 (2006), citando a Leymann, los comportamientos que pueden constituir acoso laboral son:

[...] entre otros, los siguientes: ataques verbales, insultos, ridiculización, críticas injustificadas, desacreditación profesional, amenazas constantes de despido, sobrecarga de trabajo, aislamiento social, falsos rumores, acoso sexual, no tener en cuenta problemas físicos o de salud del trabajador y hasta agresiones físicas. Las víctimas, a su vez, suelen sufrir, entre otros síntomas, los siguientes: trastornos de sueño, dolores, síntomas psicosomáticos del estrés, pérdida de memoria, crisis nerviosa, síndrome de fatiga crónica, depresión y afectación de las relaciones familiares (num. III, inc. 4, párr. 3).

\section{- Aproximación teórica al ámbito jurídico}

La OIT (2000) define el acoso laboral como la "acción verbal o psicológica de índole sistemática, repetida o persistente por la que, en el lugar de trabajo en conexión con el trabajo, una persona o un grupo de personas hiere a una víctima, la humilla, ofende o amedrenta" (p. 15). Dicha definición permite inferir que el acoso laboral comprende una gama de comportamientos ofensivos a la dignidad humana. Es por ello que los instrumentos de la OIT constituyen una fuente normativa internacional que tiene como objetivo "promover los derechos laborales, fomentar oportunidades de trabajo decente, mejorar la protección social y fortalecer el diálogo al abordar los temas relacionados con el trabajo" (OIT, 2017, párr. 3). Sin embargo, la OIT no cuenta con un instrumento jurídico específico que reglamente el acoso laboral, pero sí algunos de sus instrumentos constituyen un 
marco normativo para prevenir y combatir el acoso laboral, a saber: el convenio 155 sobre seguridad y salud en el trabajo (OIT, 1981); el convenio 111 de 1958 sobre la discriminación (empleo y ocupación) (OIT, 1958); y las recomendaciones prácticas sobre la violencia en el lugar de trabajo en sector de servicios (OIT, 2003). Finalmente, la lista de enfermedades profesionales de la OIT (2010), incluyó los riesgos psicosociales, permitiendo que el acoso laboral pueda ser considerado una enfermedad psicosocial.

A nivel local, Colombia es el único país en América Latina con una ley de alcance nacional (Morales, 2016), por lo cual se creó la Ley 1010 (2006), disposición normativa que tiene como objetivo prevenir, corregir y sancionar el acoso laboral y otros hostigamientos en el marco de las relaciones del trabajo. La Ley 1010 (2006) establece que se entenderá por acoso laboral:

Toda conducta persistente y demostrable, ejercida sobre un empleado, trabajador por parte de un empleador, un jefe o superior jerárquico inmediato o mediato, un compañero de trabajo o un subalterno, encaminada a infundir miedo, intimidación, terror y angustia, a causar perjuicio laboral, generar desmotivación en el trabajo, o inducir la renuncia de este (art. 2).

Esta normatividad gira entorno a tres pilares fundamentales: El primero permite la utilización del mecanismo de autocomposición del conflicto que genera conductas de acoso laboral, incluyendo medidas preventivas en el reglamento interno de trabajo y en el comité de convivencia laboral, tendientes a establecer escenarios de conciliación con la finalidad de erradicar o mitigar los efectos de el acoso laboral. En el segundo la víctima de manera simultanea podrá solicitar la intervención del Inspector de Trabajo, Personero Municipal, Defensoría del Pueblo, para que cesen los ataques, por parte del hostigador, que atentan contra la dignidad humana, la salud y la seguridad en el trabajo. Tercero, cuando las anteriores medidas no resulten eficaces, deberá acudir al aparato jurisdiccional en cabeza del juez laboral del lugar donde ocurrieron los hechos generadores de acoso laboral, con la finalidad de imponer la sanciones que derivan del Código Sustantivo del Trabajo. 


\section{- Normas de funcionamiento del Comité de Convivencia Laboral}

La primera instancia o herramienta para la prevención se encuentra en los reglamentos de trabajo, los cuales imponen el deber a las empresas de establecer mecanismos de prevención de acoso laboral, deber que se encuentra establecido en la Ley 1010 (2006), la cual da nacimiento a los comités de convivencia laboral.

El comité de convivencia laboral se rige por la Ley 1010 (2006), la Resolución 652 (2012) y la Resolución 1356 (2012), las cuales regulan el funcionamiento y conformación en las entidades públicas y privadas. El Comité de Convivencia Laboral tiene como finalidad prevenir el acoso laboral, al igual que previene los riesgos psicosociales derivados de este. Dada la importancia dentro de las empresas, el Comité de Convivencia Laboral se erige como el órgano que le corresponde la prevención del acoso laboral.

El Comité de Convivencia Laboral estará compuesto por dos representantes del empleador y dos de los trabajadores, con sus respectivos suplentes. Las entidades públicas y las empresas privadas podrán de acuerdo con su organización interna designar un mayor número de representantes, los cuales en todo caso son iguales en ambas partes. Elegidos por voto secreto y mediante escrutinio público. Teniendo una vigencia de dos años a partir de la fecha de su instalación. No podrán ser elegidos quienes se les haya formulado una queja de acoso laboral o que hayan sido víctimas de acoso laboral en los últimos seis meses anteriores a su conformación (Resolución 1356, 2012).

La función principal del Comité de Convivencia Laboral es recibir, tramitar las quejas interpuestas por conductas de acoso laboral que se presenten en el trabajo. Para esto, el comité deberá examinar de manera confidencial los casos puestos en conocimiento, implementando medidas preventivas y correctivas, en un espacio dialógico que deberá conllevar al ánimo conciliatorio. En ese sentido, los Comités de Convivencia Laboral tendrán un procedimiento regido por el principio de reserva o confidencialidad, autocompositivo y efectivo para erradicar las prácticas de acoso laboral. 
Al comité de convivencia laboral le está vedado determinar si una conducta es constitutiva de acoso o no, dado que tal competencia se encuentra asignada al órgano jurisdiccional, es decir al juez laboral. Por lo tanto, las decisiones del Comité son netamente preventivas y consultivas, siempre direccionadas a mecanismos auto compositivos del conflicto generado por conductas consideradas de acoso laboral.

Las competencias de los integrantes del Comité de Convivencia Laboral son:

[...] actitudinales y comportamentales tales como: respeto, imparcialidad, tolerancia, serenidad, confidencialidad, reserva en el manejo de la información, ética, habilidades de comunicación liderazgo y resolución de conflictos. Se deberá realizar capacitaciones a los miembros del comité sobre resolución de conflictos, comunicación asertiva y de temas prioritarios para el funcionamiento (Resolución 1356, 2012, art. 1, párr. 3).

El comité de convivencia laboral sesionará cada tres meses y podrá sesionar con la mitad más uno de sus integrantes, contará con facultad de reunirse extraordinariamente, si así lo requiere alguno de los integrantes.

\section{Metodología}

El tipo de investigación realizada fue descriptiva, mediante la cual se buscó "especificar propiedades, características y rasgos importantes de cualquier fenómeno que se analice" (Hernández, Fernández y Baptista, 2014, p. 119). Asimismo, se realizó la indagación de modo sistemático de las características de la población objeto de estudio, la situación de funcionalidad de los Comités de Convivencia Laboral. El enfoque de la investigación fue mixto, la etapa cuantitativa consistió en la "recolección y el análisis de datos para contestar preguntas de investigación y probar hipótesis previamente hechas, confía en la medición numérica, el conteo y frecuentemente el uso de estadística para establecer con exactitud patrones de comportamiento en una población” (Hernández et al., 2014, p. 5). Así pues, se utilizó como instrumento de recolección de información la encuesta probabilís- 
tica, cuyos resultados fueron arrojados en medición numérica, donde se utilizó el software IBM SPSS Statistics® (versión 25), para la correspondiente tabulación y análisis de la información. Igualmente, el estudio buscó comprender e interpretar la eficacia de los Comités de Convivencia Laboral en las medianas empresas del municipio de Girardot (Cundinamarca, Colombia). Lo cual permitió a través del enfoque cualitativo captar la realidad como un proceso construido socialmente por los actores en interacción.

El método de investigación utilizado fue el inductivo, el cual consiste en analizar una porción de un todo, parte de lo particular a lo general (Tamayo, 2009). Es decir, parte de hechos para realizar inferencias de carácter general de la funcionabilidad de los Comités de Convivencia Laboral y obtener la percepción de los miembros de los comités de convivencia laboral y de los trabajadores la eficacia de los comités de convivencia laboral.

Los instrumentos utilizados para el desarrollo de la investigación fueron la observación no participante, la cual consiste en adentrarse profundamente en las situaciones socio-jurídicas, con el papel activo y reflexivo de los Comités de Convivencia Laboral. Igualmente, la observación fue sistemática, estructurada, regulada o controlada. Se realizaron entrevistas semiestructuradas a las personas que conforman los Comités de Convivencia Laboral teniendo como finalidad obtener información acerca de las percepciones sobre los integrantes del Comité, así como también de los trabajadores que son representados por los integrantes del comité. Se realizó un grupo focal con el cual se aplicó el método Delphi para asegurar la conducción y conclusión acertada a partir de expertos en la materia. Por último, se utilizó una encuesta en escala Likert, la cual fue sometida al Alfa de Cronbach para medir su confiabilidad.

De otro lado, la población de esta investigación está comprendida por 46 medianas empresas del municipio de Girardot (Cundinamarca, Colombia), de acuerdo con información de la Cámara de Comercio de Girardot, Alto Magdalena y Tequendama (2016), y un total de 3200 trabajadores; la cual se tomó como población universal para la aplicación de la fórmula finita, con una probabilidad de error 
del $5 \%$ y un margen de confiabilidad del 90\%. La muestra fue de 250 trabajadores activos mayores de 18 años, de diferentes niveles y formación académica y estrato socioeconómico heterogéneo, mediante encuestas tipo Likert que arrojaron un Alfa de Cronbach de 0.820, el cual se entiende con un nivel de confiabilidad bueno.

\section{Resultados - Discusión}

De los grupos focales de las entrevistas semiestructuradas se detectaron obstáculos al funcionamiento del Comité de Convivencia Laboral que atentan contra su eficacia. A saber: dentro de los Reglamentos Internos de Trabajo no se establecen mecanismos de prevención del acoso laboral, distintos al Comité de Convivencia Laboral. El miedo a denunciar: desde la multidisciplinariedad quién lo padece sufre de temor al denunciar, a pesar de la existencia de un fuero de protección, establecido en la Resolución 2646 (2008, art. 11) del Ministerio de Trabajo, a quienes no se les podrá terminar su vinculación por el término de seis meses (Ministerio de la Protección Social, 2008). Es por eso que esta garantía del trabajador denunciante es limitada, frente a la posible intimidación o ataque que puede ejercer el victimario. La situación anterior concuerda con la encuesta tipo Likert a la población objeto de estudio.

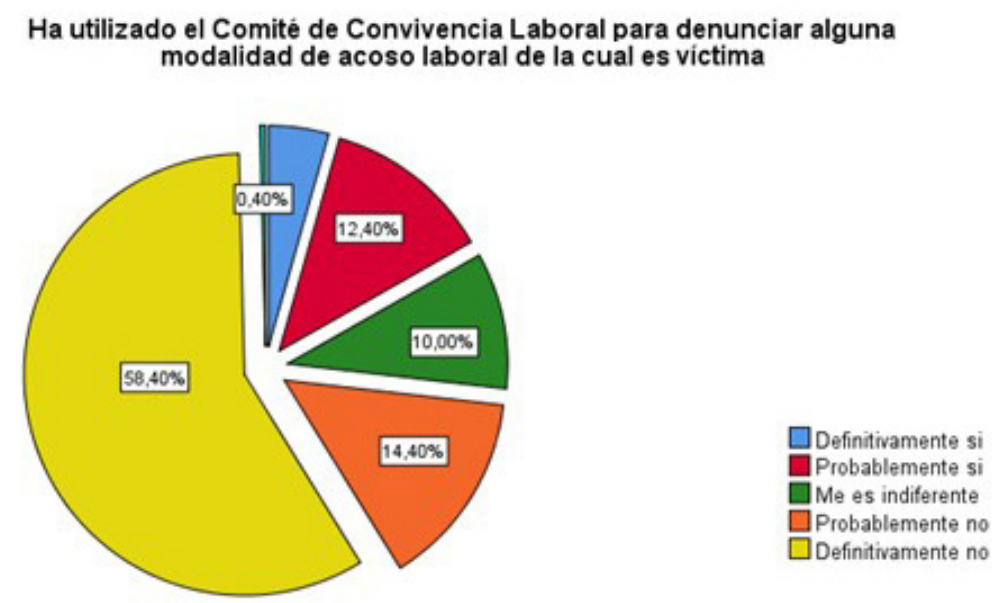

Figura 1. Utilización de los comités de convivencia laboral. Fuente: Elaboración propia. 
De la muestra objeto de estudio encuestada, el $58.4 \%$ y $14.4 \%$ no han utilizado el Comité de Convivencia Laboral, a pesar de que en las entrevistas semiestructuradas se evidencia o afirman que en algún momento de su historia laboral han padecido de acoso laboral (Figura 1). Estructurando lo afirmado por Piñuel (2001) sobre el silencio que suelen guardar las víctimas de acoso laboral, por miedo a perder el empleo.

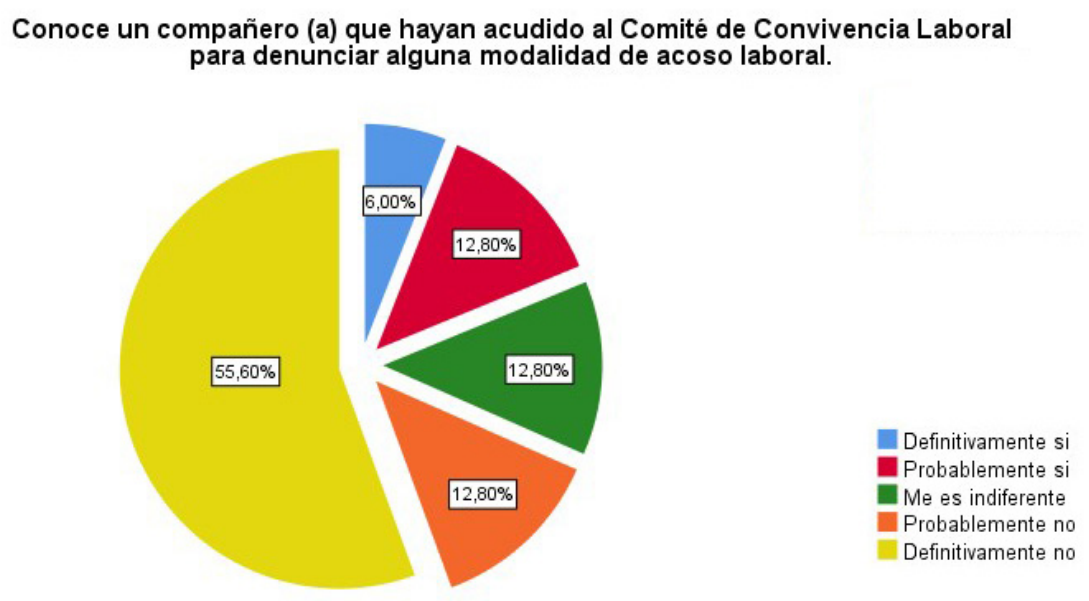

Figura 2. Denuncias modalidad de acoso laboral. Fuente: Elaboración propia.

En correlación con la Figura 1, la muestra continúa con la tendencia a no denunciar o presentar queja ante el Comité de Convivencia Laboral en un porcentaje que abarca $81.2 \%$. Lo anterior evidencia que sigue siendo un fenómeno de "opacidad" que es la invisibilización del fenómeno que se encuentra vinculado a tres aspectos: "primero, las débiles visibilizarían del fenómeno en el campo jurídico; segundo, la inexistencia de mecanismos de sanción y reparación; y tercero, a la poca relevancia que se le da al daño" (López y Seco, 2015, p. 127).

Otro elemento que le resta eficacia a los Comités de Convivencia Laboral son los medios probatorios que se aportaran al momento de instaurar la queja ya que manifiestan que este tipo de conductas son de difícil comprobación porque generalmente son de ocurrencia privada, ocultas. Es así como la Ley 1010 (2006) señala que "se presumirá que hay acoso laboral si acredita la ocurrencia repetida y pública" (art. 7). 
Ha participado en la elección de los miembros del Comité de Convivencia Laboral

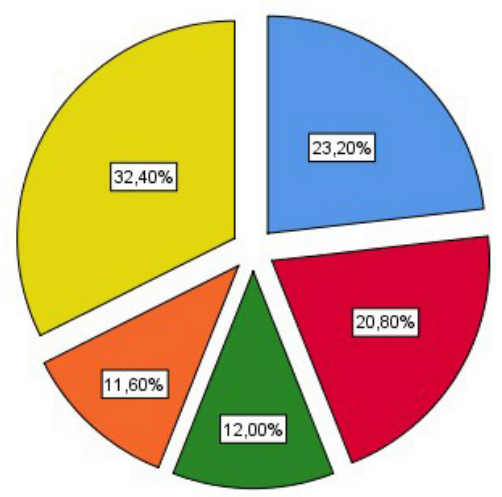

Figura 3. Elección de miembros de comité de convivencia laboral.

Fuente: Elaboración propia.

Igualmente, le resta eficacia socio-jurídica la poca imparcialidad que podría presentarse al momento de constituir el Comité de Convivencia Laboral, en el sentido que los miembros integrantes del comité son generalmente los trabajadores de confianza del victimario. Sin embargo, de acuerdo con la encuesta, el 56\% no ha participado en la elección de los miembros del Comité de Convivencia Laboral (Figura 3). Igualmente se halló en la muestra el conocimiento de la conformación de los Comités con $61.6 \%$, como lo evidencia la Figura 4.

Tiene conocimiento sobre la conformación del Comité de Convivencia Laboral de su empresa

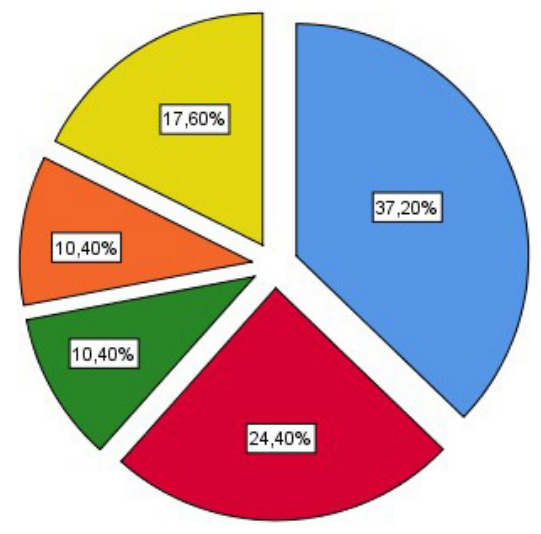

Figura 4. Conformación de comité de convivencia laboral. Fuente: Elaboración propia. 


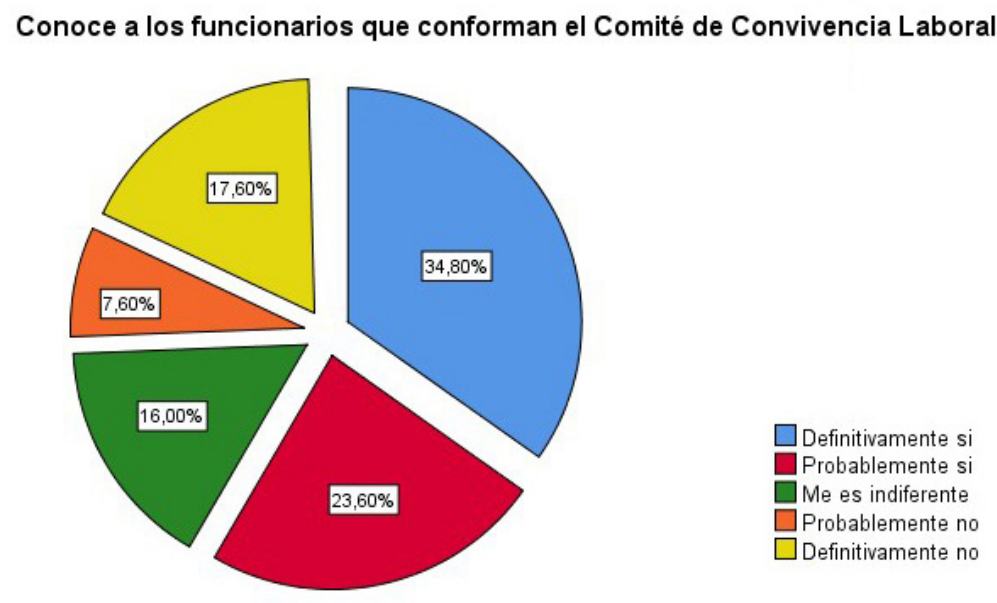

Figura 5. Conformación del comité de convivencia laboral. Fuente: Elaboración propia.

Por otra parte, el 58.4\% conoce a los funcionarios que conforman el Comité de Convivencia Laboral generando un clima de confianza al momento de iniciar el proceso conciliatorio (Figura 5). Sin embargo, en los grupos focales se afirma que puede ser contraproducente el clima de confianza en los conciliadores en el sentido que pueden ser parcializados en contra de las víctimas o viceversa con los victimarios.

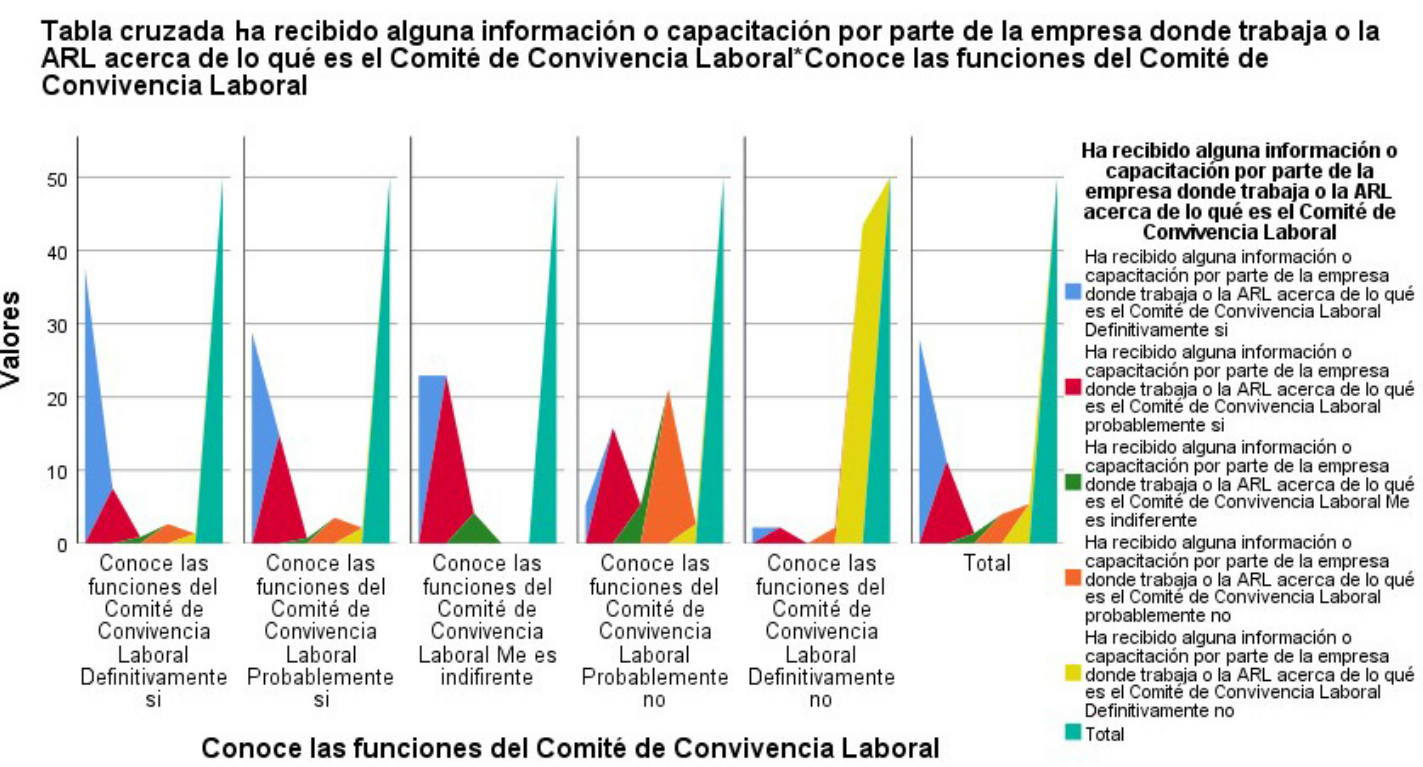

Figura 6. Funciones del comité de convivencia laboral.

Fuente: Elaboración propia. 
Frente al conocimiento de la norma con relación funciones del Comité de Convivencia Laboral (Figura 6) y la capacitación recibida acerca de los mismos, estos fueron los hallazgos que se obtuvieron al cruzar dos variables: el $85 \%$ conoce y ha recibido capitación acerca de los Comités de Convivencia Laboral. Igualmente manifiestan que no solo conocen la funcionabilidad del Comité de Convivencia Laboral, sino la normatividad relacionada con la ley de acoso laboral.

Finalmente, la legitimidad del Comité de Convivencia Laboral se ve afectada, dado que un $45 \%$ de los encuestados tienen la percepción que las decisiones adoptadas por el Comité de Convivencia Laboral favorecen al victimario o acosador. Asimismo, las decisiones que toma el Comité, según la muestra, carecen en un $65 \%$ de efectividad práctica, en el sentido que no solucionan de fondo el conflicto entre el acosador y acosado.

\section{Conclusiones}

La norma jurídica es eficaz cuando ha sido acatada produciendo la dirección deseada, es decir que la norma jurídica se cumpla u obedezca. En Colombia se ha legislado para la prevención, la corrección y la sanción de las conductas consideradas acoso laboral. En teoría, el trabajador que padece de este flagelo tiene instrumentos para enfrentar el hostigamiento psicosocial dentro del ámbito laboral. La herramienta por excelencia llamada a la prevención de este fenómeno es el Comité de Convivencia Laboral. En la práctica, este instrumento presenta poca eficacia, asociada a los obstáculos frente al funcionamiento y los resultados esperados por quienes acuden de manera primigenia a la solución del conflicto.

La poca eficacia del Comité de Convivencia Laboral en la práctica reside en el nulo o poco acompañamiento de mecanismos de prevención del acoso laboral distintos al Comité de Convivencia Laboral, dejándolo como único instrumento en el ámbito interno de las medianas empresas del municipio de Girardot. Lo anterior genera el temor a denunciar o presentar la queja, por las represarías que podrá sufrir el denunciante, situación que se refleja en 
que el $58.40 \%$ de la población objeto de estudio no ha utilizado la herramienta procesal de queja o denuncia ante el Comité de Convivencia Laboral.

Por otra parte, se halló dificultad probatoria al percibirse en los grupos focales que el recaudo probatorio es de difícil consecución por la víctima, al encontrase en un escenario de privacidad entre él, la víctima y victimario ya que el hostigamiento se produce desde una o varias personas a una persona en solitario que debido a la agresión se ve incitado a una protección inútil de la situación que tiene frente al continuo ataque (Leymann \& Gustafsson, 1996).

La investigación permite concluir la poca imparcialidad que se presenta al momento de constituir el Comité de Convivencia Laboral en el sentido que los integrantes del comité son generalmente los trabajadores de confianza del victimario. Situación que se puede presentar al momento de la elección, ya que no existe la obligación a cargo de los trabajadores designar a sus representantes en el Comité de Convivencia Laboral, "por lo tanto, no es posible obligar a ningún trabajador a que realice acciones en donde la norma le da plena libertad de acción" (Concepto 33446, 2015, párr. 5).

Igualmente, la investigación arrojó como resultado significativo que los trabajadores se encuentran capacitados en la elección, conformación y funcionamiento de los Comités de Convivencia Laboral; sin embargo, falta profundizar en capacitaciones de resolución y técnicas de conflictos, técnicas conciliatorias.

La ineficacia de los Comités de Convivencia Laboral de la población objeto de estudio genera una débil legitimación y confianza como instrumento de prevención contra el acoso laboral, considerado como un mecanismo débil al no permitir efectividad en las soluciones puestas en conocimiento al Comité al resultar en 25.4\% pocas veces efectiva. La situación anterior conlleva a que los trabajadores deban recurrir a otras instancias para denunciar este tipo de hostigamiento verbi gratia ante los inspectores de trabajo o la jurisdicción ordinaria ante el juez laboral. Infiriéndose que existe una deficiente gestión del conflicto, lo que genera un aumento del mobbing. 
En suma, normativamente el Comité de Convivencia Laboral tiene una competencia limitada, pero valiosa al momento de ser la primera herramienta que tiene la víctima para lograr cesar los hostigamientos de acoso laboral. De ahí la importancia que se articulen instrumentos distintos al Comité de Convivencia Laboral que sirvan de apoyo al momento de efectuarse una queja o denuncia con la finalidad de lograr la autocomposición del conflicto.

Por lo anterior, esta investigación consiste en una fuente de información inicial para el desarrollo de futuros estudios que profundicen en el mejoramiento del Comité de Convivencia Laboral. Del mismo modo, refuerza la importancia de implementar medidas de promoción, prevención e intervención orientadas a proteger la seguridad y la salud de los trabajadores desde los riesgos psicosociales. Asimismo, sería de relevancia la realización de estudios comparados con otros países, con la finalidad de auscultar la eficacia de las herramientas para combatir el acoso laboral dentro de las organizaciones.

\section{REFERENCIAS}

Brodsky, C. (1976). The harassed worker. Toronto: Lexington Books Heath and Company.

Calvo, R. (2007). La ineficacia de las normas jurídicas en la teoría pura del derecho. Isonomía, (27), 171-191. Disponible en https://isonomia.itam.mx/index.php/revista-cientifica/article/view/378

Cámara de Comercio de Girardot, Alto magdalena y Tequendama. (2016). Informe cuantitativo de las medianas empresas del municipio de Girardot. Girardot: Cámara de Comercio. Disponible en https://www.ccgirardot.org/

Evan, W. (1980). The Sociology of Law. New York: University of Pennsylvania.

González, J. L. (2002). El maltrato psicológico.Cómo defenderse del mobbing y otras formas deacoso. Madrid: Espasa Calpe.

Hernández, R., Fernández, C. y Baptista, L. (2014). Metodología de la investigación. México, D.F.: McGraw-Hill. 
Hirigoyen, M. (2012). El acoso moral. El maltrato psicológico en la vida cotidiana. Buenos Aires: Paidós.

Kelsen, H. (1969). Teoría general del Estado. México, D.F.: UNAM. IBM. (versión 25). IBM SPSS Statistics. [software]. New York: IBM. Disponible en https://www.ibm.com/support/pages/downloading-ibm-spss-statistics-25

Leymann, H. (1986). Vuxenmobbning - Ompsykiskt void $i$ arbetslivet. Lund: Studentlitteratur.

Leyman, H. \& Gustafsson, A. (1996). Mobbing at Work and the Development of Post-traumatic Stress Disorders. The European Journal of Work and organizational Psychology, 5(2), 251-275. http://dx.doi.org/10.1080/13594329608414858

López, C. y Seco, E. (2015). Eficacia de la ley 1010/2006 de acoso laboral en Colombia, una interpretación desde la sociología. Revista de Derecho, (44), 111-144. Disponible en https://rcientificas.uninorte.edu.co/index.php/derecho/article/view/6516

López, M. y Vásquez, P. (junio, 2007). El mobbing en las organizaciones. La ausencia de un concepto global y único. Comunicacion presentada al XXI Congreso anual, Decisiones basadas en el conocimiento y en el papel social de la empresa (Vol. 2, p. 27). AEDEM, Palma de Mallorca, España.

Lorenz, K. (1991). I am - where are yuo? The behavior of geese. Muchen: Piper.

Morales, M. (2016). Aproximación al acoso laboral desde la legislación comparada. Boletín Mexicano de Derecho Comparado, (147), 71-98. https://doi.org/10.22201/ iij.24484873e.2016.147.10640

OIT. (septiembre 10, 2017). Acerca de la OIT. [Online]. Disponible en https://www.ilo.org/global/about-the-ilo/lang--es/index.htm

OIT. (2010). Lista de enfermedades profesionales de la OIT. Ginebra: Consejo de Administración. Disponible en https://www.ilo. org/wcmsp5/groups/public/---ed_protect/---protrav/---safework/documents/publication/wcms_125164.pdf 
OIT. (2003). Repartorio de recomendaciones prácticas sobre la violencia en el lugar de trabajo en el sector de servicios y medidas para combatirla. Ginebra: Oficina Internacional del Trabajo. Obtenido de https://www.ilo.org/wcmsp5/groups/ public/---ed_protect/---protrav/---safework/documents/normativeinstrument/wcms_112578.pdf

OIT. (2000). Violencia en el trabajo. Ginebra: OIT. Recuperado de https://www.ilo.org/wcmsp5/groups/public/---ed_dialogue/--actrav/documents/publication/wcms_117581.pdf

OIT. (1981). Sobre la seguridad y salud de los trabajadores y medio ambiente. [Convenio 155]. Obtenido de https://www. ilo.org/dyn/normlex/es/f?p=NORMLEXPUB:12100:0::NO: :P12100_INSTRUMENT_ID:312300

OIT. (1958). C111 - Convenio sobre la discriminación (empleo y ocupación). [Convenio 155]. (núm. 111). Obtenido de https:// www.ilo.org/dyn/normlex/es/f?p=NORMLEXPUB:12100: 0::NO::P12100_ILO_CODE:C111

Portafolio. (agosto 26, 2018). El acoso laboral sigue creciendo en Colombia. [Online]. Disponible en https://www.mintrabajo.gov.co/web/guest/inicio

Piñuel, I. (2001). Como sobrevivir al acoso psicologico en el trabajo. Bilbao: Sal Terrae.

Ramírez, H. y Ulloa, N. (2020). Trabajo decente desde los lineamientos de la OIT: v aplicabilidad en el sector agrícola cebollero de Aquitania Boyacá. Jurídicas CUC, 16(1), 97126. https://doi.org/10.17981/juridcuc.16.1.2020.04

Remes, J., Manyika, J., Bughin, J., Woetzel, J., Mischke, J. \& Krishnan, M. (2018). Solving the Productivity Puzzle: The Role of Demandand the Promise of Digitalization. Chicago: Mckinsey Global Institute. Available from https:// www.mckinsey.com/ /media/mckinsey/featured\%20insights/meeting\%20societys $\% 20$ expectations/solving\%20 the $\% 20$ productivity $\% 20$ puzzle/mg-solving-the-productivity-puzzle--report-february-2018.pdf 
República de Colombia. Congreso de la República. (23 de enero de 2006). Por medio de la cual se adoptan medidas para prevenir, corregir y sancionar el acoso laboral. [Ley 1010]. Diario Oficial: 46160. Disponible en http://www. secretariasenado.gov.co/senado/basedoc/ley_1010_2006. html

República de Colombia. Corte Constitucional. (30 de septiembre de 2003). Exp. D-4504. [Sentencia C-873]. Magistrado Ponente: Manuel José Cepeda Espinosa. Disponible en https://www.corteconstitucional.gov.co/ relatoria/2003/c-873-03.htm

República de Colombia. Corte Constitucional. (26 de octubre de 2006). Exp. T-1377066. [Sentencia T-882]. Magistrado Ponente: Humberto Sierra Porto. Disponible en https:// www.corteconstitucional.gov.co/relatoria/2006/t-882-06. htm

República de Colombia. Ministerio de Trabajo. (2 de marzo de 2015). Comité de convivencia laboral. Desinterés en su constitución. [Concepto 33446]. Asunto: 198037. Disponible en https:/cijuf.org.co/normatividad/concepto/2015/ concepto-33446.html

República de Colombia. Ministerio de Trabajo. (18 de julio de 2012). Por medio de la cual se reglamenta la Resolución 652 de 2012. [Resolución 1356]. Diario Oficial: 48501. Disponible en https://www.icbf.gov.co/cargues/avance/ docs/resolucion_mtra_1356_2012.htm

República de Colombia. Ministerio de Trabajo. (30 de abril de 2012). Por la cual se establece la conformación y el funcionamiento del Comité de Convivencia Laboral en las entidades públicas y privadas y se dictan otras disposiciones. [Resolución 652]. Diario Oficial: 48427. Disponible en https://secretariageneral.gov.co/transparencia/marco-legal/normatividad/resoluci\%C3\%B3n-652-2012-1 
República de Colombia. Ministerio de la Protección Social de Colombia. (17 de julio de 2008). Por la cual se establecen disposiciones y se definen responsabilidades para la identificación, evaluación, prevención, intervención y monitoreo permanente de la exposición a factores de riesgo psicosocial en el trabajo y para la determinación del origen de las patologías causadas por el estrés ocupacional. [Resolución 2646]. Diario Oficial: 47059. Disponible en https://www. icbf.gov.co/cargues/avance/docs/resolucion_minproteccion_2646_2008.htm

Schwab, K. (2016). La Cuarta Revolución Industrial. Barcelona: Debate. Recuperado de http://40.70.207.114/documentosV2/ La\%20cuarta\%20revolucion\%20industrial-Klaus\%20 Schwab\%20(1).pdf

Tamayo, M. (2009). El proceso de la investigación científica. México, D.F.: Limusa.

Artículo resultado de la investigación "Efectividad de los Comités de Convivencia Laboral en las medianas empresas del municipio de Girardot- Cundinamarca" Investigación patrocinada por la Dirección Nacional de Investigaciones de la Corporación Universitaria Uniminuto.

Julián Andrés Gaitán Reyes es Magíster en Derecho de la Universidad Sergio Arboleda (Colombia). Especialista en Derecho procesal de la Universidad Libre (Colombia). Abogado de la Universidad Cooperativa de Colombia. Integrante del grupo de investigación Multiverso de la Seguridad Laboral, línea de investigación Gestión del riesgo/amenazas de la Corporación Universitaria Uniminuto (Colombia). Investigador Asociado de Minciencias. https://orcid. org/0000-0003- 0072-1548 
Luperly Palacio Rodríguez es Magíster en prevención de riesgos laborales de Universidad Internacional de la Rioja (España). Especialista en derecho laboral y la seguridad social de la Universidad Libre (Colombia). Abogada de la Universidad Cooperativa de Colombia. Profesional en Salud Ocupacional de la Universidad del Tolima (Colombia). Integrante del grupo de investigación Multiverso de la Seguridad Laboral. Integrante del grupo de investigación Multiverso de la Seguridad Laboral, línea de investigación Gestión del riesgo/ amenazas de la Corporación Universitaria Uniminuto (Colombia). https://orcid.org/0000-0002-4862-8054 